INPLASY

PROTOCOL

To cite: Huang et al. Herbal acupoint sticking versus acupuncture in the treatment of cervical spondylosis: A systematic review and metaanalysis. Inplasy protocol 202170022. doi: 10.37766/inplasy2021.7.0022

Received: 09 July 2021

Published: 09 July 2021

Corresponding author: Huang Xin

13077923191@163.com

Author Affiliation:

Affiliated Hospital of Jiangxi University of Traditional Chinese Medicine; Graduate School of Jiangxi University of Traditional Chinese Medicine.

Support: JX Education

Department.

Review Stage at time of this submission: Completed but not published.

Conflicts of interest:

None declared.

\section{Herbal acupoint sticking versus acupuncture in the treatment of cervical spondylosis: A systematic review and meta-analysis}

\author{
Huang, X1; Zhang, YY²; Liu, YL³ ; Bao, JW4; Liu, X5; \\ Chen, YW6; Xia, ZC7; Wang, L8.
}

Review question / Objective: Evaluation of the efficacy and safety of acupressure compared to acupuncture in the treatment of cervical spondylosis from randomized controlled trials.

Eligibility criteria: (1)Study subjects: Patients who were diagnosed with reference to the diagnostic criteria of the Guidelines for the Diagnosis, Treatment and Rehabilitation of Cervical Spondylosis or the diagnostic criteria of the Diagnostic Efficacy Criteria of Chinese Medical Evidence regarding cervical spondylosis or the diagnosis of cervical spondylosis symptoms plus imaging examination. (2) Interventions: only the observation group used acupoint dressing alone or acupoint dressing combined with acupuncture, and the control group used acupuncture alone without any additional treatment modalities. Translated with http://www.DeepL.com/Translator (free version).

INPLASY registration number: This protocol was registered with the International Platform of Registered Systematic Review and Meta-Analysis Protocols (INPLASY) on 09 July 2021 and was last updated on 09 July 2021 (registration number INPLASY202170022).

\section{INTRODUCTION}

Review question / Objective: Evaluation of the efficacy and safety of acupressure compared to acupuncture in the treatment of cervical spondylosis from randomized controlled trials.

Condition being studied: Cervical spondylosis is a common and prevalent disease in the middle-aged and elderly 
population and is a secondary lesion caused by degenerative changes in the cervical disc and the structural involvement of the tissue surrounding its adjacent structures. Epidemiological surveys have shown that approximately $80 \%$ to $90 \%$ of people present with disc degeneration on magnetic resonance imaging (MRI) at age 50 years. The peak incidence in Chinese men and women is between the ages of 40 and 60 years. The incidence of cervical spondylosis is increasing yearly and at a younger age due to technological advances in society, changes in lifestyle and an increase in the number of workers. A review of the global burden of low back and neck pain estimated that more than one third of the global population suffers from mechanical neck pain lasting at least 3 months, emphasizing the global health impact of whiplash. Treatment of cervical spondylosis can be surgical or conservative. Conservative treatment is the treatment of choice for most patients, and many conservative treatment measures have been applied for cervical spondylosis, including nonsteroidal anti-inflammatory drugs (NSAIDs), epidural steroid injections, acupuncture, and acupressure. The use of TCM external treatment is one of the characteristics of TCM orthopedics and traumatology, in which acupoint dressing, which involves the identification of drugs and acupoints for individual patient characteristics, is a commonly used TCM external treatment method and has been widely used in clinical trials in recent years. Recent studies have shown that acupuncture point application has significant effects in reducing chronic pain in the neck through the stimulation of herbal meridians, and is increasingly popular among patients for its unique advantages such as convenience, effectiveness, and painlessness. After a preliminary search and database analysis, we found that the volume of literature on randomized controlled trials of acupressure and acupuncture for cervical spondylosis is increasing year by year. Previous clinical trials have shown that acupressure and acupuncture can reduce pain and improve the quality of life of patients. These effects persist in patients with cervical spondylosis. However, the current level of evidence-based medicine remains inadequate due to the limited size and sample size of clinical centers. Therefore, this article will conduct a two-pronged Meta-analysis, Meta-analysis I: Efficacy and safety of acupressure combined with acupuncture group compared to acupuncture alone group for the treatment of cervical spondylosis; Meta-analysis II: Efficacy and safety of acupressure group compared to acupuncture group for the treatment of cervical spondylosis. The efficacy and safety of acupressure and acupuncture in the treatment of cervical spondylosis were compared with the clinical protocol of acupuncture, which has been recognized as safe and effective and widely used, to provide a sufficient evidence-based medical basis for the clinical application of acupressure.

\section{METHODS}

Search strategy: The search time interval was from the creation of each database to May 15, 2021, using a combination of keyword and free-word searches. Search terms included "acupressure," "acupuncture," and "cervical spondylosis. The search terms in the English database included "acupuncture", "Acupoint Application Therapy", "cervical spondylosis", etc.

Participant or population: Patients who were diagnosed with reference to the diagnostic criteria of the Guidelines for the Diagnosis, Treatment and Rehabilitation of Cervical Spondylosis or the diagnostic criteria of the Diagnostic Efficacy Criteria of Chinese Medical Evidence regarding cervical spondylosis or the diagnosis of cervical spondylosis symptoms plus imaging examination.

Intervention: Herbal acupoint sticking.

Comparator: Acupuncture.

Study designs to be included: Randomized controlled trials (RCTs) will be included. 
Eligibility criteria: (1)Study subjects: Patients who were diagnosed with reference to the diagnostic criteria of the Guidelines for the Diagnosis, Treatment and Rehabilitation of Cervical Spondylosis or the diagnostic criteria of the Diagnostic Efficacy Criteria of Chinese Medical Evidence regarding cervical spondylosis or the diagnosis of cervical spondylosis symptoms plus imaging examination. (2) Interventions: only the observation group used acupoint dressing alone or acupoint dressing combined with acupuncture, and the control group used acupuncture alone without any additional treatment modalities. Translated with http:// www.DeepL.com/Translator (free version).

Information sources: Computer searches of China Knowledge Network ( CNKI) (1979-), Wanfang Digital Journal Network (WANFANG DATA) (1990-), China Biomedical Literature Database ( CBM) (1978-), Vipers Database ( VIP) (1989-), Cochrane library, EMbase ( 1974-), PubMed (1950-).

Main outcome(s): Total effective rate, clinical cure rate, visual analog scale (VAS), numerical rating scale (NRS), and NPQ neck pain scale.

Quality assessment / Risk of bias analysis: As for the quality assessment of the literature, we will have 2 researchers assess the quality and risk of bias of all included literature using the Cochrane recommended risk of bias assessment tool. In case of inconsistent assessment, a third researcher will be consulted and discussed to deal with it. Inverted funnel plot of publication bias for total efficiency comparisons to determine Risk of bias analysis.

Strategy of data synthesis: 1. Data extraction and management. Literature data extraction was done independently by 2 researchers, filling out a data sheet developed by the researchers in a unified manner. The content of data extraction included: (1). General information: article title, first author, corresponding author, time of published study, evaluation communication, and contact information. (2). Study method: design model, sample size, random assignment, random hiding, blinding, baseline level. (3). Participants: patient age, gender, diagnostic criteria for cervical spondylosis, severity. (4). Interventions: acupoint application, acupuncture, acupuncture points, duration of treatment, frequency of treatment. (5). Efficacy evaluation: total effective rate, clinical cure rate, visual analog scale (VAS), numerical rating scale (NRS), NPQ neck pain scale, and number of adverse reactions. 2. Statistical analysis Metaanalysis for this study will be performed using RevMan 5.4 and Stata 16.0 statistical software. The study was tested for heterogeneity using REM (random effects model) or FEM (fixed effects model) with the test criteria of FEM if $\mathrm{I}^{2}<50 \%$ and REM if $I^{2} \geq 50 \%$. if the heterogeneity between results was too large, sensitivity analysis was used to infer the source of heterogeneity and analyze it. Second, the dominance ratios of binary categorical variables and their $95 \%$ confidence intervals were used as effect analysis indicators according to different types of statistics. For continuous variables, standardized mean differences and their $95 \%$ confidence intervals were used as effect analysis indicators. Forest plots and inverted funnel plots were drawn using RevMan 5.4 and Stata 16.0 software, and potential publication bias was analyzed by inverted funnel plots.

Subgroup analysis: 1. The total effective rate, clinical healing rate, VAS score, NRS score and NPQ neck pain scale score of the acupoint application group compared with the acupoint application combined with acupuncture group. 2. The total effective rate, clinical recovery rate, VAS score of the acupressure group compared with the acupuncture group.

Sensitivity analysis: Sensitivity analysis was performed using both the replacement model and the article-by-article exclusion method, and if the results obtained were 
not reversed, it indicated that the Metaanalysis results were stable.

Country(ies) involved: China.

Keywords: Herbal acupoint sticking; Acupuncture; Cervical spondylosis; Meta analysis; Randomized controlled trial.

Contributions of each author:

Author 1 - Huang Xin.

Author 2 - Zhang Yaoyao.

Author 3 - Liu Yuling.

Author 4 - Bao Jiewei.

Author 5 - Liu Xiang.

Author 6 - Chen Yuwen.

Author 7 - Xia Zicheng.

Author 8 - Wang Li. 